\title{
Exploring dark sector parameters in light of neutron star temperatures
}

\author{
Guey-Lin Lin $\oplus^{1, *}$ and Yen-Hsun Lin $\circledast^{1,2, \dagger}$ \\ ${ }^{1}$ Institute of Physics, National Yang Ming Chiao Tung University, Hsinchu 30010, Taiwan \\ ${ }^{2}$ Institute of Physics, Academia Sinica, Taipei 11529, Taiwan
}

(Received 27 February 2021; accepted 12 August 2021; published 15 September 2021)

\begin{abstract}
Using neutron stars (NS) as a dark matter (DM) probe has gained broad attention recently; either from heating due to DM annihilation or its stability under the presence of DM. In this work, we investigate spin-1/2 fermionic DM $\chi$ charged under the $U(1)_{X}$ in the dark sector. The massive gauge boson $V$ of $U(1)_{X}$ gauge group can be produced in NS via DM annihilation. The produced gauge boson can decay into Standard Model (SM) particles before it exits the NS, despite its tiny couplings to SM particles. Thus, we perform a systematic study on $\chi \bar{\chi} \rightarrow 2 V \rightarrow 4 \mathrm{SM}$ as a new heating mechanism for NS in addition to $\chi \bar{\chi} \rightarrow 2 \mathrm{SM}$ and kinetic heating from DM-baryon scattering. The self-trapping due to $\chi V$ scattering is also considered. We assume the general framework that both kinetic and mass mixing terms between $V$ and SM gauge bosons are present. This allows both vector and axial-vector couplings between $V$ and SM fermions even for $m_{V} \ll m_{Z}$. Notably, the contribution from axial-vector coupling is not negligible when particles scatter relativistically. We point out that the above approaches to DM-induced NS heating are not yet adopted in recent analyses. Detectabilities of the aforementioned effects to the NS surface temperature by the future telescopes are discussed as well.
\end{abstract}

DOI: 10.1103/PhysRevD.104.063021

\section{INTRODUCTION}

It has been widely accepted that one-fifth of the total energy of the Universe consists of dark matter (DM). Though multidisciplinary strategies are employed to identify its essence, either from direct [1-10] or indirect detections [11-17], the nature of DM remains a puzzle. The approach of using neutron stars (NS) as the DM probe has been proposed from the heating effect due to DM [1830], the NS instability caused by DM gravitational collapse [29,31-42], and gravitation wave emitted from the merger of binary NS admixed with DM [43-45]. Novel ways of constraining long-lived particles through the NS in the Milky Way was also investigated recently [46]. In addition, DM self-interaction naturally arises in various phenomenological models and was proposed to resolve many issues in the small-scale structure, e.g., core-cusp, missing satellite, too-big-to-fail, and diverse galactic rotation curve (see Ref. [47] for a review). Current astrophysical observations constrain DM self-interaction cross section $\sigma_{\chi \chi}$ in the range [48-52]

\footnotetext{
"glin@nycu.edu.tw

yenhsun@gate.sinica.edu.tw
}

Published by the American Physical Society under the terms of the Creative Commons Attribution 4.0 International license. Further distribution of this work must maintain attribution to the author(s) and the published article's title, journal citation, and DOI. Funded by SCOAP.

$$
0.1 \mathrm{~cm}^{2} \mathrm{~g}^{-1} \leq \sigma_{\chi \chi} / m_{\chi} \leq 10 \mathrm{~cm}^{2} \mathrm{~g}^{-1}
$$

where $m_{\chi}$ is the DM mass.

DM self-interaction can be understood phenomenologically as an exchange of the $U(1)_{X}$ gauge boson $V$ between dark matter particles. Assuming DM $\chi$ is a spin- $1 / 2$ fermion carrying $U(1)_{X}$ dark charge $g_{d}$, its interaction with $V$ is given by the Lagrangian

$$
\mathcal{L}_{\mathrm{DM}}=i \bar{\chi} \gamma^{\mu}\left(\partial_{\mu}-i g_{d} V_{\mu}\right) \chi-m_{\chi} \bar{\chi} \chi,
$$

where the associated DM self-interaction cross section $\sigma_{\chi x}$ can be calculated from $\mathcal{L}_{\mathrm{DM}}$ and constrained by Eq. (1). Here we consider the scenario of symmetric dark matter [53] where the numbers $\chi$ and $\bar{\chi}$ are identical. It has been proposed that the vector boson $V$ in the dark sector can mix with SM photons and $Z$ bosons through kinetic [54-59] and mass mixing terms [60-62]; the latter generally arise from extended Higgs sectors. Ref. [61] provides an example by introducing a two Higgs doublet $\Phi_{1}, \Phi_{2}$, with $\Phi_{1}$ coupled to SM fermions, and one scalar Higgs singlet $\phi$. Both $\Phi_{2}$ and $\phi$ carry the dark charge $g_{d}$ and the mixing among $\Phi_{1}$, $\Phi_{2}$, and $\phi$ are neglected for simplicity. Hence neither $\Phi_{2}$ nor $\phi$ couples to SM fermions. The vacuum expectation values of Higgs scalars give mass terms for $Z, V$, and their mixing, which, together with kinetic mixing terms, are given by 


$$
\begin{array}{r}
\mathcal{L}_{\text {gauge }}=-\frac{1}{4} B_{\mu \nu} B^{\mu \nu}+\frac{1}{2} \frac{\varepsilon_{\gamma}}{\cos \theta_{W}} B_{\mu \nu} V^{\mu \nu}-\frac{1}{4} V_{\mu \nu} V^{\mu \nu}, \\
\mathcal{L}_{\text {mass }}=\frac{1}{2} m_{Z}^{2} Z_{\mu} Z^{\mu}-\varepsilon_{Z} m_{Z}^{2} Z_{\mu} V^{\mu}+\frac{1}{2} m_{V}^{2} V_{\mu} V^{\mu},
\end{array}
$$

where $B^{\mu \nu} \equiv \partial_{\mu} B_{\nu}-\partial_{\nu} B_{\mu}$ is the $U(1)_{Y}$ field strength in SM while $\varepsilon_{\gamma}$ and $\varepsilon_{Z}$ are the kinetic and $V-Z$ mass mixing parameters respectively. It is important to note that we do not invoke spontaneous symmetry breaking for generating DM mass as can be seen from Eq. (2) [63]. Since none of the scalar fields mentioned above couple to $\chi$, the only mediator between the dark and visible sector is the dark boson $V$. Explicitly speaking, the electromagnetic (EM) and neutral-current (NC) interactions between $V$ and SM fermions $f$ resulting from mixing terms in Eqs. (3) and (4) are given by

$$
\mathcal{L}_{\mathrm{DS}-\mathrm{SM}}=\left(\varepsilon_{\gamma} e J_{\mu}^{\mathrm{EM}}+\tilde{\varepsilon}_{Z} \frac{g_{2}}{\cos \theta_{W}} J_{\mu}^{\mathrm{NC}}\right) V^{\mu},
$$

where $g_{2}$ is the $S U(2)_{L}$ coupling and $J_{\mu}^{\mathrm{EM}}$ and $J_{\mu}^{\mathrm{NC}}$ are the SM electromagnetic and neutral currents, respectively. The coefficient $\tilde{\varepsilon}_{Z}$ is a linear combination of two mixing parameters and it reduces to $\varepsilon_{Z}$ for $m_{V} \ll m_{Z}$. Its general expression is given in Appendix A.

In this paper, we examine the effect of DM heating due to the above phenomenological setup for a nearby three gigayear-old (Gyr-old) and isolated NS. The associated temperature is around $100 \mathrm{~K}$ according to the standard cooling mechanism if there is no other heating source. Therefore, any temperature deviation from this benchmark value can be potentially due to DM annihilation in the star. DM annihilation channels in this regard include not only $\chi \bar{\chi} \rightarrow$ $f \bar{f}$ but also $\chi \bar{\chi} \rightarrow 2 V$, provided $m_{V}<m_{\chi}$ and the decay length of $V \rightarrow f \bar{f}$ is smaller than the radius of the star. Since $V$ couples to neutral scalar bosons to acquire its own mass, the annihilation process $\chi \bar{\chi} \rightarrow V^{*} \rightarrow V+s$ (with $s$ one of the neutral scalar bosons) is also possible for $m_{V}+m_{s}<2 m_{\chi}$. Such a process yields a comparable heating effect to that given by $\chi \bar{\chi} \rightarrow 2 V$ but involves an additional mass parameter $m_{s}$. For simplicity in our discussions, we shall not consider this kinematic region. We refer the readers to Refs. $[64,65]$ for the phenomenology of such an annihilation channel.

Searching the nearby old and cold NS can improve our understanding about DM. The new dynamics emerging from the above phenomenological setup will be discussed in the following sections. For completeness, we also analyze the signal to noise ratio (SNR) in the James Webb Space Telescope (JWST) [66]. Future telescopes such as European Extremely Large Telescope (E-ELT) and Thirty-Meter Telescope (TMT) [67] will constrain DM properties with unprecedented sensitivities. In the following sections, we employ the NS mass $M_{0}=1.4 M_{\odot}$ and and the radius $R_{0}=12 \mathrm{~km}$. We also replace $g_{d}$ with $\alpha_{\chi}=$ $g_{d}^{2} / 4 \pi$ and all equations are expressed in terms of natural units $\hbar=c=k_{B}=1$.

\section{DM CAPTURE AND NS TEMPERATURE}

When a NS swipes through space, the DM particles in the halo can scatter with the baryons and leptons inside the star. Once DM loses an appreciable fraction of kinetic energy, it will be gravitationally captured by the NS. This capture process has been investigated extensively with contributions from neutrons, protons, and leptons as well as relativistic corrections included in Refs. $[68,69]$. In this paper, only the neutron contribution to the capture rate $C_{c}$ is considered. Contributions from other particle species are ignored due to their small yields. The DM number $N_{\chi}$ in the star satisfies the differential equation

$$
\frac{d N_{\chi}}{d t}=C_{c}-C_{a} N_{\chi} N_{\bar{\chi}}
$$

while the anti-DM number $N_{\bar{\chi}}$ evolves according to

$$
\frac{d N_{\bar{\chi}}}{d t}=C_{c}-C_{a} N_{\bar{\chi}} N_{\chi}
$$

Here $C_{a}$ is the DM annihilation rate. Both coefficients $C_{c}$ and $C_{a}$ are well studied and the expressions can be found in Refs. $[23,68,69]$ and references therein. We do not reproduce them here. Thus, the exact solutions to Eqs. (6) and (7) are obtained

$$
N_{\chi}=N_{\bar{\chi}}=C_{c} \tau_{\mathrm{eq}} \tanh \left(\frac{t}{\tau_{\mathrm{eq}}}\right),
$$

where $\tau_{\mathrm{eq}}=1 / \sqrt{C_{c} C_{a}}$ is the equilibrium timescale. Once $t>\tau_{\text {eq }}, d N_{\chi} / d t=0$, and $N_{\chi}\left(t>\tau_{\text {eq }}\right)=\sqrt{C_{c} / C_{a}}$ according to Eq. (6). The total annihilation rate at this stage only depends on the capture rate since $\Gamma_{a}=C_{a} N_{\chi} N_{\bar{\chi}}=C_{c}$. ${ }^{1}$ Note that $C_{c}$ depends on $\sigma_{\chi n}$, and $\sigma_{\chi n} \leq \sigma_{\chi n}^{\text {geom }} \approx 10^{-44} \mathrm{~cm}^{2}$ where $\sigma_{\chi n}^{\text {geom }}$ is the geometric cross section. In principle, the maximum capture rate is determined by

\footnotetext{
${ }^{1}$ The $C_{a}$ depends on the annihilation cross section $\langle\sigma v\rangle$ explicitly but its effect only appears in the total annihilation rate $\Gamma_{A}$ through $\tanh \left(t / \tau_{\mathrm{eq}}\right)$ as $\tau_{\mathrm{eq}}^{2} \propto 1 /\langle\sigma v\rangle$. No matter what the value of $\langle\sigma v\rangle$ is, one always has $\tanh \left(t / \tau_{\mathrm{eq}}\right) \approx 1$ as long as the NS age is greater than the equilibrium time scale. In this case the total annihilation rate $\Gamma_{A}$ no longer depends on $\langle\sigma v\rangle$, but rather it is solely determined by the capture rate $C_{c}$. We have carefully examined that even $\langle\sigma v\rangle$ is an order of magnitude smaller than the thermal relic one given in Ref. [70], the NS can still attain equilibrium at $t \sim \mathrm{Gyr}$ and have no sensitivity on $\langle\sigma v\rangle$ anymore. The typical timescale for $\tau_{\mathrm{eq}}$ is about hundreds to thousands of years for $\left(\langle\sigma v\rangle, \sigma_{\chi n}\right)=\left(6 \times 10^{-26} \mathrm{~cm}^{3} \mathrm{~s}^{-1}, 10^{-47} \mathrm{~cm}^{2}\right)$ for $m_{\chi}$ around $\mathrm{MeV}$ to $\mathrm{TeV}$.
} 
$C_{c}\left(\sigma_{\chi n}^{\mathrm{geom}}\right)=C_{c}^{\mathrm{geom}}$. Besides, when DM falls into the NS surface, it is accelerated up to $0.3 c-0.5 c$. The nonrelativistic (NR) limit for calculating $\sigma_{\chi n}$ is not applicable. Furthermore one has to consider contributions from axialvector coupling due to $V-Z$ mass mixing given by $\mathcal{L}_{\text {mass }}$. We have thoroughly included these effects. A brief discussion on how to compute $\sigma_{\chi^{n}}$ in terms of relativistic kinematics is given in Appendix A.

NS is known to suffer from eternal cooling due to neutrino and photon emissions. Without an extra energy injection, the NS temperature drops until it releases all its heat. However, if SM particles are produced due to DM annihilation in the star, these particles can become a heat source and potentially prevent the star from inevitable cooling. Therefore, the evolution of the NS interior temperature $T_{b}$ is governed by the equation

$$
\frac{d T_{b}}{d t}=\frac{-\epsilon_{\nu}-\epsilon_{\gamma}+\epsilon_{\chi}}{c_{V}},
$$

where $\epsilon_{\nu} \approx 2.1 \times 10^{4} \mathrm{erg} \mathrm{cm}^{-3} \mathrm{~s}^{-1}\left(T_{b} / 10^{7} \mathrm{~K}\right)^{8}$ is the neutrino emissivity, $\epsilon_{\gamma} \approx 1.8 \times 10^{14} \mathrm{ergcm}^{-3} \mathrm{~s}^{-1}\left(T_{b} / 10^{8} \mathrm{~K}\right)^{2.2}$ is the photon emissivity, $\epsilon_{\chi}$ is the DM emissivity that is responsible for the heating from DM annihilation, and $c_{V}$ the NS heat capacity [18]. Additionally, the surface temperature $T_{s}$ observed by a distant observer is related to $T_{b}$ by $T_{s} \approx 8.7 \times 10^{5} \mathrm{~K}\left(g_{s} / 10^{14} \mathrm{~cm} \mathrm{~s}^{-1}\right)^{1 / 4}\left(T_{b} / 10^{8} \mathrm{~K}\right)^{0.55}$

where $g_{s}=G M / R^{2} \approx 1.85 \times 10^{14} \mathrm{~cm} \mathrm{~s}^{-2}$ accounts for the redshift correction from the surface gravity of the star. It is also pointed out that when $T_{b}<3700 \mathrm{~K}$, there is no distinction between $T_{b}$ and $T_{s}$ [23].

During each annihilation, a pair of DMs release $2 m_{\chi}$ of energy in the form of SM particles or dark bosons depending on which channels are kinematically allowed. The total energy release rate by DM is $\mathcal{E}_{\chi}=2 m_{\chi} \Gamma_{a} \sum_{i} b_{i}$ where $b_{i}$ is the branching ratio of a specific channel, e.g., $e^{ \pm}, \mu^{ \pm}, \tau^{ \pm}$or $q \bar{q}$, and $\sum_{i} b_{i} \leq 1$. Neutrino pair $\nu \bar{\nu}$ is also part of the annihilation channel in the presence of $V-Z$ mass mixing, but it cannot contribute to the heating. In addition to the annihilation, DM also loses kinetic energy $E_{k}$ to the star through the capturing process. This has been realized as the kinetic heating [21] with the rate $\mathcal{K}_{\chi}=C_{c} E_{k}=C_{c} m_{\chi}(\gamma-1)$, where $\gamma=1 / \sqrt{1-v^{2}}$ is the Lorentz factor. ${ }^{2}$ Thus, DM emissivity $\epsilon_{\chi}$ is given by

$$
\epsilon_{\chi}=\frac{\mathcal{E}_{\chi}+\mathcal{K}_{\chi}}{V}
$$

where $V$ is the NS volume.

\footnotetext{
${ }^{2}$ Even if DM is not captured, energy deposition still occurs as long as $\chi n$ scattering can happen. On the other hand, the kinetic heating effect from such uncaptured DM is relatively small and negligible in our calculation.
}

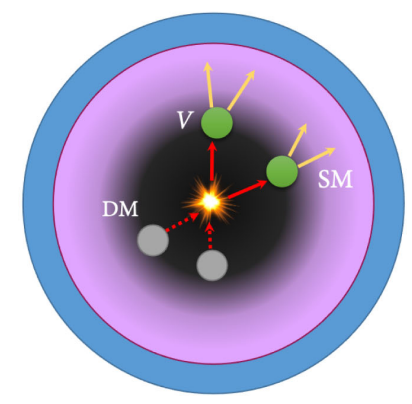

(a)

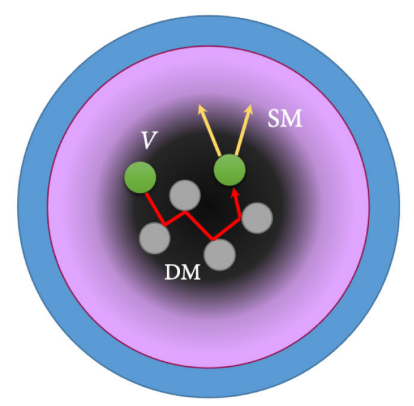

(b)
FIG. 1. DM heating from dark boson production $\chi \bar{\chi} \rightarrow 2 V$. Left: $V$ decays into SM particles before it exits the star. Right: $V$ is self-trapped due to multiple $\chi V$ scatterings and then decays.

\section{DECAYS OF DARK BOSON}

Here we discuss the case of $V$ produced by DM annihilation. $V$ is usually produced in DM rich environment. If $V$ can subsequently scatter off the surrounding DM multiple times, it could lose energy and be selftrapped. It then decays promptly as shown in Fig. 1(b). However, such self-trapping effect is in general inefficient since the $\chi V$ scattering length $\ell_{\chi V}$ is much larger than the thermal radius $r_{\text {th }}$. Hence the scattering rate is suppressed and irrelevant to the heating. We show detailed discussions in Appendix C. Another trapping is due to the scattering between $V$ and neutrons. On the other hand the relevant cross section is further suppressed by the factor $\tilde{\varepsilon}_{Z}^{4}$ and the scattering length is expected to be much larger than the NS radius. It is safe to omit this effect in our calculation as well.

However, $V$ can decay into other SM particles before it propagates to the surface as long as the decay length $\ell_{\mathrm{dec}}$ is shorter than $R_{0}$ (see Fig. 1(a)). The decay length is given by $\ell_{\mathrm{dec}}=v \gamma \tau_{\mathrm{dec}}$ with $v \equiv \sqrt{1-m_{V}^{2} / m_{\chi}^{2}}$ the velocity of $V$, and $\tau_{\text {dec }} \equiv \Gamma_{V}^{-1}$ the lifetime of $V$ at rest, where $\Gamma_{V}$ is the total decay width. Since $V$ is produced on shell, we do not consider $V$ decaying back to $\chi$ due to $m_{V}<m_{\chi}$. The probability for $V$ to convert into SM particles after a propagation distance $r$ is

$$
F=1-e^{-r / \ell_{\mathrm{dec}}}
$$

We took $r=R_{0}$ in the calculation. However, if neutrino is the decay product, it cannot be considered as the heating source and must be subtracted. By examining the numerical results for $F$, we found that $V$ can decay before it exits the star in most of our parameter space of interest. This implies that $\chi \bar{\chi} \rightarrow 2 V$ also plays an important role in NS heating (see Appendix $\mathrm{C}$ for details). Generally speaking, NS contain muons and electrons that are degenerate. To enable the decay $V \rightarrow \ell \bar{\ell}$ with $\ell$ corresponding to either the electron or the muon, $V$ should not only be heavier than 

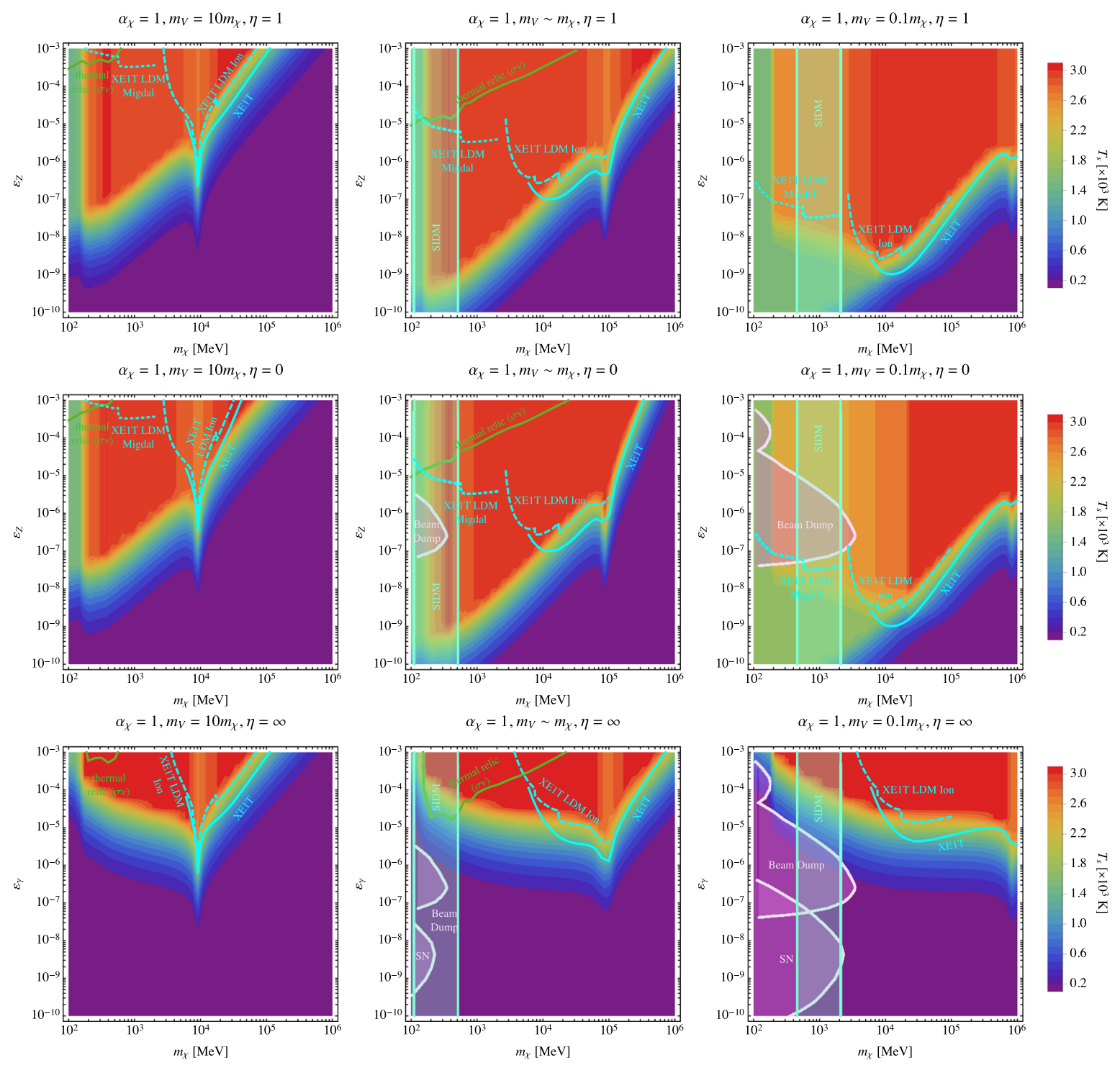

FIG. 2. NS surface temperature $T_{s}$ in the $m_{\chi}-\varepsilon$ plane. We took the age of NS is 3 Gyrs and the lowest $T_{s}=100 \mathrm{~K}$ without DM heating. All figures have $\alpha_{\chi}=1$ and $\eta=\varepsilon_{\gamma} / \varepsilon_{Z}$. From top to bottom, $\eta=1,0$, and $\infty$. From left to right, $m_{V} / m_{\chi}=10,1,0.1$. Various constraints from XENON1T [8], XENON LDM [9,10], SIDM [48-52], SN1987A [74,75], beam dump experiments [76-78], and the parameter curve rendering thermal relic cross section are shown as well.

$2 m_{\ell}$, it also has to be energetic enough so that the kinetic energy of $l$ exceeds the chemical potential of $l$ for preventing the Pauli blocking effect. This condition has been implemented in our study.

Given the information in this section, we summarize that even when $\chi \bar{\chi} \rightarrow 2 V$ dominates the annihilation channel for $m_{V}<m_{\chi}$, the heating effect is still efficient due to $V$ decays. However, the self-trapping is generally unimportant due to $\ell_{\chi V} \gg r_{\text {th }}$ in this paper.

\section{IMPLICATION OF DM ON NS TEMPERATURE}

In this section, we describe how NS surface temperature $T_{s}$ is affected by the DM annihilation. If $\epsilon_{\chi}$ is negligible, the standard cooling mechanism gives $T_{s} \approx 100 \mathrm{~K}$ for a
3-Gyr-old NS. But when $\epsilon_{\chi}$ is large enough to counterbalance $\epsilon_{\gamma, \nu}, T_{s}$ could remain at a relatively higher temperature. We present the numerical results of $T_{s}$ for both $\alpha_{\chi}=1$ and 0.01 in Figs. 2 and 3 respectively. The adjacent DM density around NS is assumed to be the same as that of the solar system, $\rho_{\chi}=0.3 \mathrm{GeV} / \mathrm{cm}^{3}$, since we aim for the nearby isolated NS. The DM mass scale is shown from $100 \mathrm{MeV}$ to $10^{6} \mathrm{MeV}$. Once $m_{\chi} \lesssim 100 \mathrm{MeV}$, all of the annihilation channels to fermions will be Pauli blocked except neutrinos. Nonetheless, there is no upper limit for DM mass in NS. But heavier $m_{\chi}$ results in lesser DM number density which makes the NS sensitivity worse. In addition, Refs. [71-73] pointed out when $m_{\chi} \gtrsim \mathcal{O}(10-100) \mathrm{TeV}$, it requires multiple scatterings to capture the DM and implies 

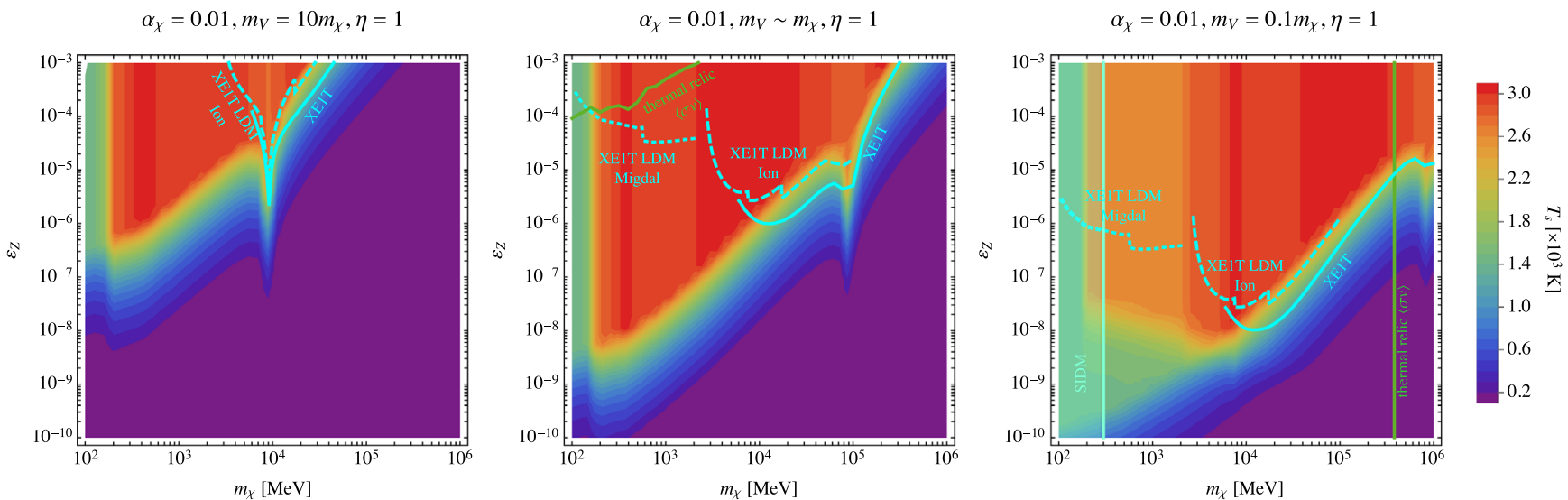

$\alpha_{\chi}=0.01, m_{V}=10 m_{\chi}, \eta=0$

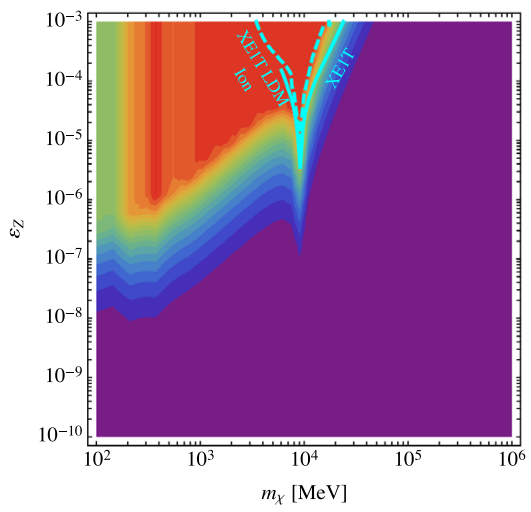

$\alpha_{\chi}=0.01, m_{V} \sim m_{\chi}, \eta=0$

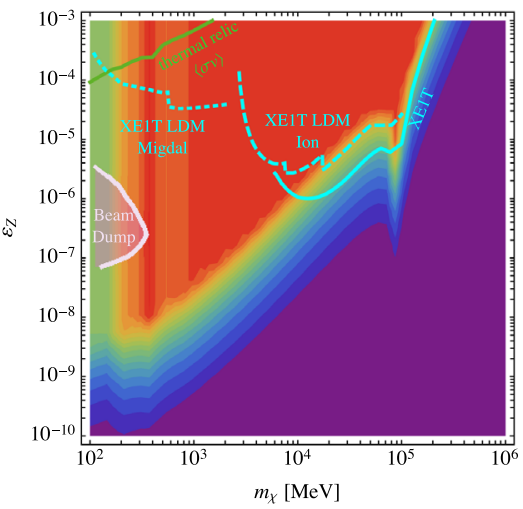

$\alpha_{\chi}=0.01, m_{V}=0.1 m_{\chi}, \eta=0$

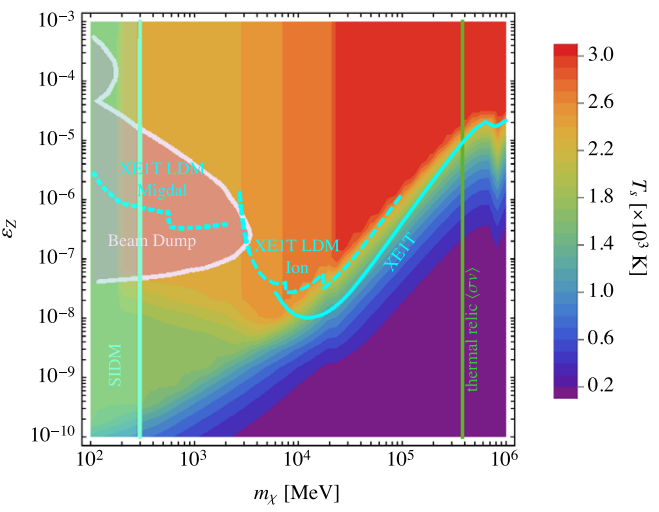

$\alpha_{\chi}=0.01, m_{V} \sim m_{\chi}, \eta=\infty$
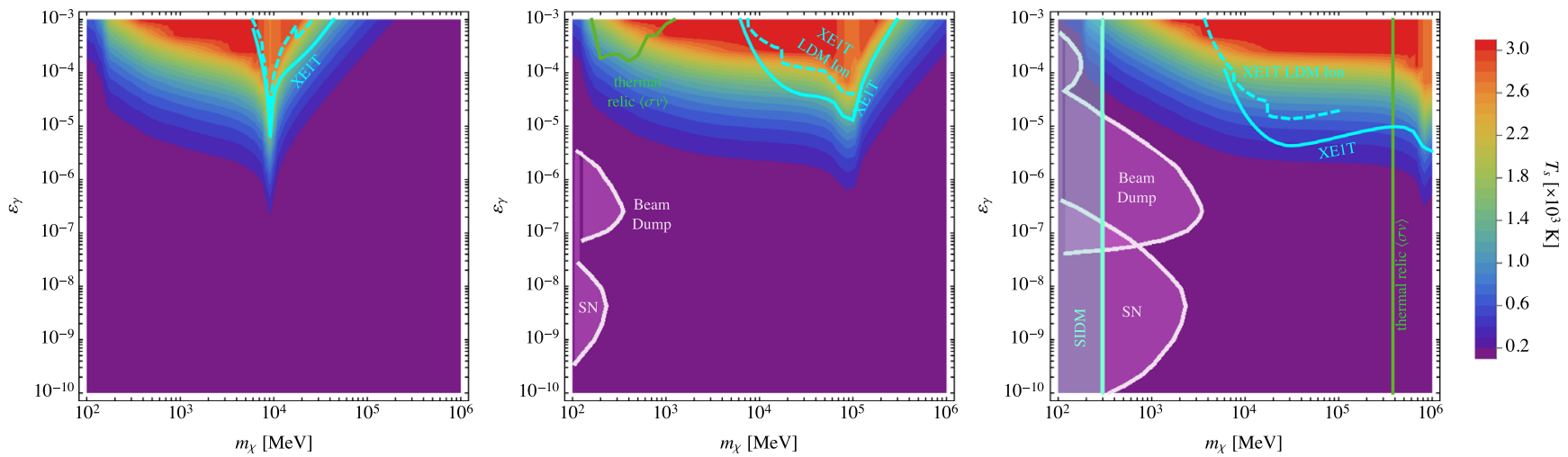

FIG. 3. The same as Fig. 2 except $\alpha_{\chi}=0.01$.

that the single-scattering capture is inefficient. Thus, we restrict our discussion below to the TeV DM where NS has better sensitivity and can be complementary to current DM direct searches.

In the following, we discuss the general trends of the numerical results in terms of $\alpha_{\chi}=1$, (see Fig. 2) unless specified otherwise. The values of $\sigma_{\chi n}$ and $\langle\sigma v\rangle$ are computed with the rest of the parameters $\left(m_{\chi}, m_{V}, \epsilon_{\gamma}\right.$, $\left.\epsilon_{Z}\right)$ taking the values shown on each figure. Constraints on these parameters according to the thermal relic density and direct searches are displayed as well. The conclusions can be applied to $\alpha_{\chi}=0.01$ directly (see Fig. 3). A simple understanding on $\alpha_{\chi}$ is that the dark sector interactions are proportional to $\alpha_{\chi}^{2}$ and DM-SM interactions are proportional to $\alpha_{\chi}$. The derivations of such features on the scattering cross sections for all interactions are given in the appendixes.

The values for the parameter $\eta \equiv \varepsilon_{\gamma} / \varepsilon_{Z}$ from top to bottom are 1 (combined, $\varepsilon_{Z}=\varepsilon_{\gamma} \neq 0$ ), 0 (pure $V-Z$ mixing, $\varepsilon_{\gamma}=0$ ) and $\infty$ (pure kinetic mixing, $\varepsilon_{Z}=0$ ), respectively. From left to right, we have $m_{V} / m_{\chi}=10$ (heavy mediator), 1 (equal mass) and 0.1 (light mediator). $T_{s}$ is indicated by the color bar placed on the right and the lowest temperature is $100 \mathrm{~K}$. Without annihilation, e.g., no 
anti-DM exists, solely kinetic heating can raise $T_{s}$ up to $1750 \mathrm{~K}$. If DM annihilation is included, $T_{s}$ can maximally reach to $3100 \mathrm{~K}$.

Various constraints are also plotted, including XENON1T [8], XENON LDM (low mass DM) based on the ionization [10], and of Migdal [9] effects, SIDM [48-52], SN1987A [74] and beam dump experiments [7678]. The parameter curve rendering DM annihilation cross section at the thermal relic value [70] in the early Universe is plotted in green on each figure for comparison. ${ }^{3} \mathrm{We}$ adopted the method given in Ref. [79] for computing the Sommerfeld enhancement factor. The DM relative velocity in the early Universe is taken to be $c / 3$. (See Appendix B for details.) Here we present the thermal relic cross section as a reference point and refer the readers to Refs. $[53,58,80]$ for detailed discussions. In addition, although the captured DMs can have a relatively large Sommerfeld enhancement due to low velocities, ${ }^{4}$ the enhanced $\langle\sigma v\rangle$ only shortens the equilibrium timescale $\tau_{\text {eq }}$. When $t \gg \tau_{\text {eq }}$, the total annihilation rate only depends on the capture rate with $\Gamma_{A}=C_{c}$. The NS is generally insensitive to the Sommerfeld enhancement as long as the DM is in equilibrium.

\section{A. Case for $\boldsymbol{m}_{V} \geq \boldsymbol{m}_{\chi}$}

When $m_{V} \geq m_{\chi}$, only $\chi \bar{\chi} \rightarrow f \bar{f}$ is allowed. A dip occurs on each plot in Fig. 2 with this mass ordering. The resonant point is caused by the pole in $\tilde{\varepsilon}_{Z}$ given by Eq. (A5) when $m_{V}=m_{Z}$ with $m_{Z}$ the SM $Z$ boson mass. In fact the value for $\tilde{\varepsilon}_{Z}$ at this point is $-i\left(\varepsilon_{Z}+\varepsilon_{\gamma} \tan \theta_{W}\right) m_{Z} / \Gamma_{Z}$, which is enhanced by the factor $m_{Z} / \Gamma_{Z}$.

Thus, the DM-neutron scattering cross section $\sigma_{\chi n}$ depends on $\tilde{\varepsilon}_{Z}$ and is proportional to

$$
\sigma_{\chi n} \propto \frac{\alpha_{\chi} \tilde{\varepsilon}_{Z}^{2}}{m_{V}^{4}} \frac{m_{\chi}^{2} m_{n}^{2}}{\left(m_{\chi}+m_{n}\right)^{2}} \min (\xi, 1)
$$

in the NR limit. (See Eq. (A12) for reference.) ${ }^{5}$ The last term shows the suppression factor due to Pauli blocking where $\xi \sim q / \mu_{F}$ with $q$ being the momentum transfer during the scattering and $\mu_{F}$ is the neutron chemical potential.

\footnotetext{
${ }^{3}$ Since we have taken DM as Dirac fermions in the symmetric scenario [53], the thermal relic cross section is therefore two times larger than that in the Majorana DM case. Note that the thermal relic $\langle\sigma v\rangle$ is in general $m_{\gamma}$ dependent and slightly deviates from the canonical value $6 \times 10^{26} \mathrm{~cm}^{3} \mathrm{~s}^{-1}$ [70]. We have incorporated this for determining the green curve on each figure.

${ }^{4}$ Assuming DMs are thermalized with the NS core where $T_{\chi}=T_{b}$. Thus the mean velocity is about $\sqrt{T_{\chi} / m_{\chi}}$.

${ }^{5}$ In the numerical calculation, we used the general expression for $\sigma_{\chi n}$, Eq. (A7), and the derivation is given in the same appendix. Nonetheless, Eq. (12), or Eq. (A12), is simpler and suitable for our discussions in the main text.
}

In the equilibrium epoch, $t \gg \tau_{\text {eq }}$, the total annihilation rate $\Gamma_{A}=C_{c} \propto \sigma_{\chi n}{ }^{6}$. When $\tilde{\varepsilon}_{Z}$ is at the resonant point, $\sigma_{\chi n}$ is enhanced drastically by the factor $m_{Z}^{2} / \Gamma_{Z}^{2}$ so does the DM heating resulted from DM emissivity $\epsilon_{\chi}$. This accounts for the dip at $m_{V}=m_{Z}$ in each figure.

On the other hand, DM heating for $m_{\chi}$ in the sub-GeV region is much stronger. It can be understood that, as $m_{\chi} \ll m_{n}, q \propto m_{\chi}$ while $m_{V} / m_{\chi}$ is held fixed, we have $\sigma_{\chi n} \propto \tilde{\varepsilon}_{Z}^{2} / m_{\chi}$ according to Eq. (12). Hence a smaller $m_{\chi}$ leads to a larger $\sigma_{\chi n}$ as well as a more effective DM heating. However, the effect of DM heating will not grow indefinitely with $\tilde{\varepsilon}_{Z}$ as $\sigma_{\chi n} \leq \sigma_{\chi n}^{\text {geom }} \approx 10^{-44} \mathrm{~cm}^{2}$. The maximum $T_{s}$ caused by DM heating saturates when $\sigma_{\chi n}=\sigma_{\chi n}^{\text {geom }}$ and is around $3100 \mathrm{~K}$. This justifies our numerical results in Fig. 2 that $T_{s}$ does not increase further when $\tilde{\varepsilon}_{Z}$ is sufficiently large for a given $m_{\chi}$.

For all plots in Fig. 2, DM heating becomes weaker instead of proportional to $1 / m_{\chi}$ for $m_{\chi} \lesssim \mathcal{O}(170) \mathrm{MeV}$. Although DM is capable of producing $e^{ \pm}$and $\mu^{ \pm}$in this mass range, the chemical potentials for both particles are $\mu_{F}^{e} \sim \mathcal{O}(170) \mathrm{MeV}$ and $\mu_{F}^{\mu} \sim \mathcal{O}(70) \mathrm{MeV}$. All channels are Pauli blocked and only pions formed by $q \bar{q}$ are allowed until $m_{\chi}<m_{\pi}$. Nonetheless, in the presence of $V-Z$ mass mixing, neutrinos are also part of the annihilation products and take a significant branching ratio in the DM annihilation in such a mass region. Neutrinos cannot contribute to the heating - this explains why $T_{s}$ is much colder when $m_{\chi} \lesssim \mathcal{O}(170) \mathrm{MeV}$. The $\mathrm{DM}$ heating in this region is mainly due to kinetic heating. As $\sigma_{\chi n}=\sigma_{\chi n}^{\text {geom }}$, the resulted $T_{s}$ is around $1750 \mathrm{~K}$ from pure kinetic heating.

Various $\eta$ values in Fig. 2 characterize the contributions from $\varepsilon_{\gamma, Z}$ to $\tilde{\varepsilon}_{Z} \cdot{ }^{7}$ Both $\eta=1$ and 0 are similar because even when $\varepsilon_{\gamma} \neq 0$, its effect on $\tilde{\varepsilon}_{Z}$ is suppressed by $m_{V}^{2} / m_{Z}^{2}$ as seen from Eq. (A5). For $\eta=1$, the kinetic mixing can contribute comparably to the $V-Z$ mass mixing unless $m_{V}>m_{Z}$. This can be clearly seen in Fig. 2 that the difference between $\eta=1$ and 0 is apparent only in $m_{V}>$ $m_{Z}$ region, which is the region to the right of the dip. To the left of the dip, the contribution from $\varepsilon_{\gamma}$ to $\tilde{\varepsilon}_{Z}$ for $\eta=1$ is negligible.

For $\eta=\infty, \varepsilon_{Z}$ vanishes so that the only contribution to $\tilde{\varepsilon}_{Z}$ comes from $\varepsilon_{\gamma}$. As discussed earlier, the effect of the kinetic mixing term is suppressed by $m_{V}^{2} / m_{Z}^{2}$ and thus $\sigma_{\chi n} \propto \tilde{\varepsilon}_{Z}^{2} \propto \varepsilon_{\gamma}^{2} m_{V}^{4} / m_{Z}^{4}$. The associated DM heating is, in

\footnotetext{
${ }^{6} \mathrm{We}$ found that the equilibrium condition holds in most of the parameter space in this work. However, in the calculation we adopted $\Gamma_{A}=C_{a} N_{\chi} N_{\bar{\chi}}$ with $N_{\chi}\left(N_{\bar{\chi}}\right)$ given by Eq. (8), instead of simply assuming $\Gamma_{A}=C_{c}$.

${ }^{7}$ Since neutron charge is neutral, $Q=0$, the effect of kinetic mixing $Q \varepsilon_{\gamma}$ in Eq. (A4a) has zero contribution to $\sigma_{\chi n}$. Hence $\sigma_{\chi n} \propto \tilde{\varepsilon}_{Z}^{2}$. Nonetheless, if protons in the NS are considered, then $\varepsilon_{\gamma}$ contributes to the DM-proton cross section $\sigma_{\chi p}$ as a consequence of nonvanishing $Q \varepsilon_{\gamma}$.
} 
general, much weaker than the cases with $\eta=1$ and 0 . However, the advantage of $\eta=\infty$ is that no neutrinos can be produced by the DM annihilation due to the absence of $V-Z$ mass mixing. The energy released from DM annihilation can be fully deposited into the NS. This accounts for the higher $T_{s}$ than when $\eta=1$ and 0 in terms of the same $\sigma_{\chi n}$, but the difference is not apparent. Numerical calculation shows it is around ten to $\mathcal{O}(100) \mathrm{K}$.

\section{B. Case for $\boldsymbol{m}_{V}<\boldsymbol{m}_{\chi}$}

For the light mediator case, the channel $\chi \bar{\chi} \rightarrow 2 V$ dominates over $\chi \bar{\chi} \rightarrow f \bar{f}$ due to $\alpha_{\chi} \gg \varepsilon_{\gamma, Z}$ in general as long as $F \sim 1, V$ can fully decay into SM particles before it exits the NS. The resulting heating from $\chi \bar{\chi} \rightarrow 2 V$ with $V \rightarrow f \bar{f}$ can be appreciable as shown in the rightmost panel of Fig. 2. The heating region in the $m_{V}<m_{\chi}$ case is much more expanded than the $m_{V} \gg m_{\chi}$ case since lighter $m_{V}$ induces larger $\sigma_{\chi n}$, as shown in Eq. (12). The resulting effects from different $\eta \mathrm{s}$ are similar to those in the previous subsection. When DM mainly annihilates to $2 V$, the thermal relic cross section is controlled by $\alpha_{\chi}$ and $m_{\chi}$ while it is independent of $\varepsilon_{\gamma, Z}$. Hence the thermal relic cross section only constrains $m_{\chi}$ when $\alpha_{\chi}$ and $m_{V}$ are fixed. For $\alpha_{\chi}=1$ and 0.01 , the $m_{\chi}$ values rendering the thermal relic cross section are around $2 \times 10^{8} \mathrm{MeV}$ and $5 \times 10^{5} \mathrm{MeV}$, respectively.

\section{DETECTABILITY OF THE FUTURE TELESCOPE}

Since DM annihilation could significantly affect the NS surface temperature $T_{s}$, we discuss the detectability of $T_{s}$ in the JWST and similar telescopes in the future. The blackbody spectral flux density with $T_{s}$ at a given frequency $\nu$ is given by [21]

$$
\begin{aligned}
f_{\nu}\left(\nu, T_{s}, d\right) & =\frac{4 \pi^{2} \nu^{3}}{e^{2 \pi \nu / k_{B} T_{s}}-1}\left(\frac{R_{0} \gamma}{d}\right)^{2} \\
& =\frac{\left(k_{B} T_{s}\right)^{3}}{2 \pi}\left(\frac{a^{3}}{e^{a}-1}\right)\left(\frac{R_{0} \gamma}{d}\right)^{2},
\end{aligned}
$$

where $R_{0}$ is the NS radius, $\gamma \approx 1.35$ is the relativistic factor of DM on the NS surface, $d$ is the distance between the NS and the Earth, and $a=\omega / k_{B} T_{s}$. It is easy to estimate that $f_{\nu}$ peaks at $a \approx 3$ and the peak value is clearly proportional to $T_{s}^{3}$. Taking $T_{s}=2000 \mathrm{~K}$ and $d=10 \mathrm{pc}$ as an example, $f_{\nu}$ peaks at $\nu^{-1}=2 \mu \mathrm{m}$ with the peak value of $0.84 \mathrm{nJy}$. The SNR for JWST-like telescope scales as $\sqrt{t_{\text {exp }}}$ for a given $\nu$ where $t_{\exp }$ is the exposure time. This scaling stems from the fact that, for the exposure time $t_{\text {exp }}$, the signal photon number in the frequency range $[\nu, \nu+d \nu]$ is proportional to $f_{\nu} \cdot d \nu \times t_{\text {exp }}$. On the other hand, the statistical fluctuation of the photon number, arising mainly

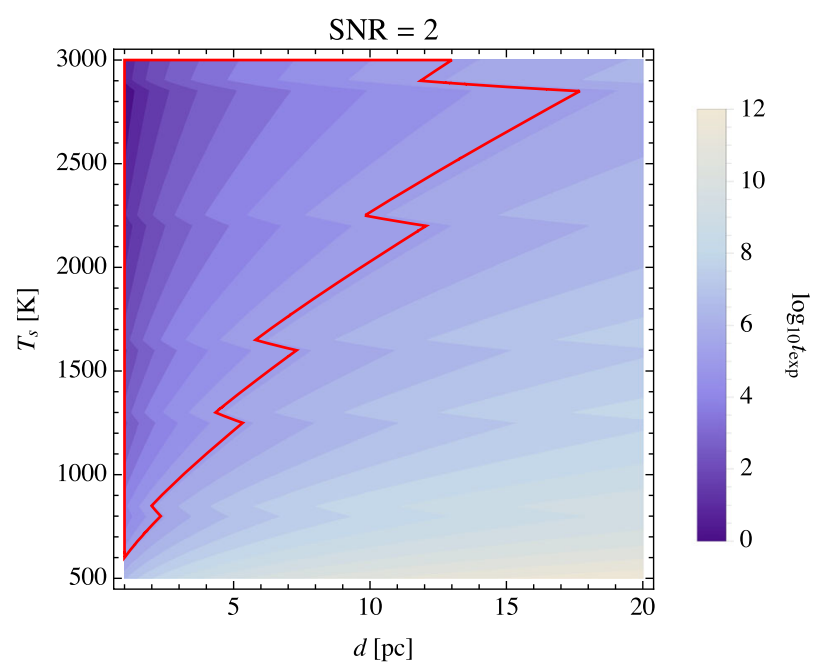

FIG. 4. The exposure time $t_{\exp }$ for $\mathrm{SNR}=2$ in JWST. The region enclosed by the red line indicates $t_{\exp } \leq 10^{5} \mathrm{~s}$.

from in-field and scattered zodiacal light, scattered thermal emission from the telescope, and the scattered starlight, scales as $\sqrt{t_{\exp }}$ for a given frequency $\nu$. This results into the above mentioned scaling for SNR. It is important to note that SNR is a complicated function of $\nu$ because the above mentioned backgrounds are also wavelength dependent [66].

As reported in Ref. [81], JWST covers $0.8 \mu \mathrm{m}$ to $5.0 \mu \mathrm{m}$ imaging sensitivity in its Near-Infrared Imager and Slitless Spectrograph (NIRISS) with multiple filters. For example the F200W filter centered at $\nu^{-1}=2 \mu \mathrm{m}$ can reach SNR $=$ 10 with $f_{\nu}=10 \mathrm{nJy}$ and $t_{\text {exp }}=10^{4} \mathrm{~s}$. To reach the peak of $f_{\nu}$ for $T_{s}=2000 \mathrm{~K}$, i.e., $0.84 \mathrm{nJy}$ with $\mathrm{SNR}=10$, one requires $1.4 \times 10^{6} \mathrm{~s}$ of exposure time. For $\mathrm{SNR}=2$, which is the criterion for our presentation below, the required exposure time is $5.6 \times 10^{4} \mathrm{~s}$.

In Fig. 4, we plot the $t_{\text {exp }}$ for obtaining SNR $=2$ over $d-T_{s}$ plane. The region enclosed by the red line represents $t_{\text {exp }}<10^{5} \mathrm{~s}$. There are multiple filters available for NIRI with $\nu^{-1}$ centered at various different values [81]. We select the filter with $\nu^{-1}$ most suitably matching the corresponding blackbody wavelength at $T_{s}$. For instance, the F200W filter is used for $T_{s}$ close to $2000 \mathrm{~K}$, while the $\mathrm{F} 277 \mathrm{~W}$ filter is adopted for $T_{s}$ around $1500 \mathrm{~K}$. The switching of filters when appropriate is reflected in the zigzag behavior of the red sensitivity curve in Fig. 4. In principle, as $\sigma_{\chi n} \sim \sigma_{\chi n}^{\text {geom }}$, kinetic heating can maximally warm the NS up to $1750 \mathrm{~K}$ without DM annihilation. For NS that is located within $10 \mathrm{pc}$, JWST can achieve SNR $=2$ with $t_{\exp } \leq 10^{5} \mathrm{~s}$ for $T_{s} \geq 1750 \mathrm{~K}$

\section{SUMMARY AND OUTLOOK}

In this work we have investigated the new dynamics arising from the kinetic mixing and $V-Z$ mass mixing 
between the dark gauge boson $V$ of the broken $U(1)_{X}$ symmetry and neutral gauge bosons in SM. In particular, $V-Z$ mass mixing induces a resonance at $m_{V} \approx m_{Z}$, which can be seen from the pole of $\tilde{\varepsilon}_{Z}$ at $m_{V}=m_{Z}$. The axialvector part of the coupling between $V$ and SM fermions has been included in our calculations. As $\chi \bar{\chi} \rightarrow 2 V$ dominates the annihilation channel for $m_{V}<m_{\chi}, V$ can decay into a pair of SM fermions before it exits NS and induces NS heating in addition to $\chi \bar{\chi} \rightarrow f \bar{f}$. Although this contribution appears naturally in the dark boson model considered here, it is usually not included in the model-independent analysis, such as the one performed in Ref. [23]. We also demonstrated numerically that NS can provide constraints on sub-GeV DM with feeble coupling to SM particles complementary to the current direct search. The detectability with reasonable $t_{\exp }$ in JWST telescopes is discussed. Similar conclusion can be drawn for the future JWST-like telescopes.

We note that this work only considers $\chi n$ scattering in the capture rate. This explains why NS is not sensitive to the dark sector when $\varepsilon_{Z}=0(\eta=\infty)$. Neutrons interact with $\mathrm{DM}$ only through $\mathrm{NC}$ interaction governed by $\tilde{\varepsilon}_{Z}$. Once $\varepsilon_{Z}=0$, NC interaction becomes suppressed since $\varepsilon_{\gamma}$ in $\tilde{\varepsilon}_{Z}$ is oppressed by $m_{V}^{2} / m_{Z}^{2}$. However, NS also consists of protons, although the fraction of them is rather small. When protons are included, charged-current interaction will be involved for the capture of DM and NS remains sensitive to the dark sector even for $\varepsilon_{Z}=0$. In general, NS sensitivity will be improved by including proton contributions. We leave this for future studies.

\section{ACKNOWLEDGMENTS}

G. L. L. is supported by the Ministry of Science and Technology, Taiwan under Grant No. 107-2119-M-009017-MY3. Y. H. L. is supported by the Postdoctoral Scholar Program of the Academia Sinica, Taiwan.

\section{APPENDIX A: DM-NEUTRON INTERACTION}

When DM falls into NS, they could scatter with neutrons via exchanging the dark boson $V$ as shown in Fig. 5. The kinetic mixing and $V-Z$ mass mixing generate vector and axial-vector interactions between $V$ and SM fermions. The usual derivation of these interactions proceeds through the diagonalization of both $\mathcal{L}_{\text {gauge }}$ and $\mathcal{L}_{\text {mass }}$ in Eqs. (3) and (4), which gives rise to relations between fields in the gauge basis and those in mass eigenstate basis. However, since we are only interested in interactions up to $\mathcal{O}\left(\varepsilon_{\gamma}\right)$ or $\mathcal{O}\left(\varepsilon_{Z}\right)$, we do not need to perform the diagonalization but rather treating the mixing terms $\varepsilon_{\gamma} B_{\mu \nu} V^{\mu \nu} /\left(2 \cos \theta_{W}\right)$ and $\varepsilon_{Z} m_{Z}^{2} Z_{\mu} V^{\mu}$ as perturbations. These two mixing terms generate the following two-point functions at the tree level

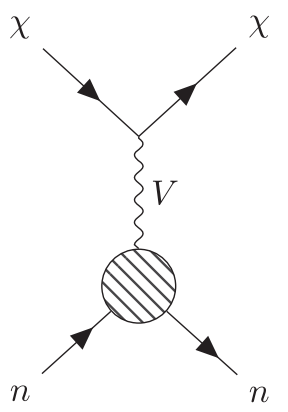

FIG. 5. Feynman diagram for DM-neutron scattering. The blob is an effective vertex that includes both vector and axial-vector contributions from kinetic mixing and $V-Z$ mass mixing.

$$
\begin{aligned}
i \Pi_{V \gamma}^{\mu \nu} & =i \varepsilon_{\gamma} k^{2} g^{\mu \nu}, \\
i \Pi_{V Z}^{\mu \nu} & =-i\left(\varepsilon_{\gamma} \tan \theta_{W} k^{2}+\varepsilon_{Z} m_{Z}^{2}\right) g^{\mu \nu},
\end{aligned}
$$

where $k$ is the four-momentum of $V$ entering into kinetic mixing or $V-Z$ mixing vertex. Hence the electromagnetic coupling of $V$ to SM fermions results from multiplying the two-point function $i \Pi_{V \gamma}^{\mu \nu}$, the photon propagator $i D_{\alpha \mu}^{\gamma}(k)$, and the electromagnetic coupling $i e A_{\alpha} J_{\mathrm{EM}}^{\alpha}$, as shown in Fig. 6. This multiplication leads to

$$
i e J_{\mathrm{EM}}^{\alpha} \frac{-i g_{\alpha \mu}}{k^{2}} i \varepsilon_{\gamma} k^{2} g^{\mu \nu} V_{\nu}=i e \varepsilon_{\gamma} J_{\mathrm{EM}}^{\nu} V_{\nu} .
$$

Similarly, the NC coupling of $V$ to SM fermions is given by multiplying the two-point function $i \Pi_{V Z}^{\mu \nu}$, the $Z$ boson propagator $i D_{\alpha \mu}^{Z}(k)$, and the $\mathrm{NC}$ coupling $i g Z_{\alpha} J_{\mathrm{NC}}^{\alpha} / \cos \theta_{W}$. This gives rise to

$$
\begin{aligned}
& \frac{i g}{\cos \theta_{W}} J_{\mathrm{NC}}^{\alpha} \frac{-i}{k^{2}-m_{Z}^{2}+i m_{Z} \Gamma_{Z}}\left(g_{\alpha \mu}-\frac{k_{\alpha} k_{\mu}}{m_{Z}^{2}}\right) \\
& \quad \times(-i)\left(\varepsilon_{\gamma} \tan \theta_{W} k^{2}+\varepsilon_{Z} m_{Z}^{2}\right) g^{\mu \nu} V_{\nu} \\
& =\frac{-i g}{\cos \theta_{W}} J_{\mathrm{NC}}^{\nu} V_{\nu} \frac{\left(\varepsilon_{\gamma} \tan \theta_{W} m_{V}^{2}+\varepsilon_{Z} m_{Z}^{2}\right)}{\left(m_{V}^{2}-m_{Z}^{2}+i m_{Z} \Gamma_{Z}\right)} .
\end{aligned}
$$

Here we have used the physical conditions $k^{2}=m_{V}^{2}$ and $k_{\mu} \epsilon_{V}^{\mu}=0$. We have also chosen the unitary gauge for the

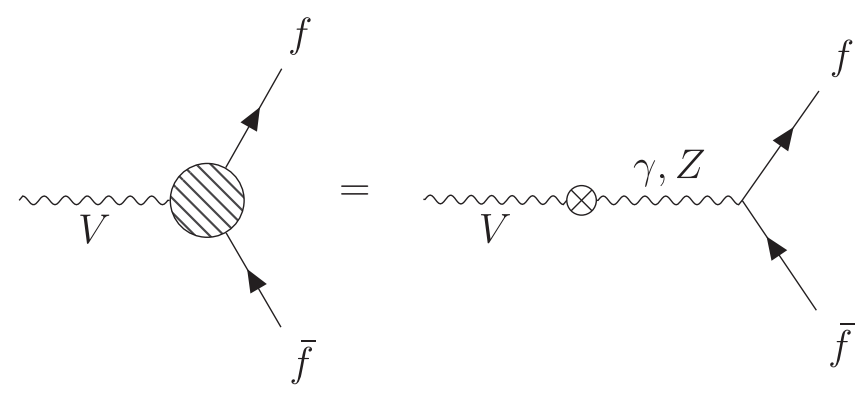

FIG. 6. Feynman diagrams contributing to the coupling of dark boson $V$ to SM fermions. 
TABLE I. Values of $Q$ and $I_{3}$ for quarks, leptons, and neutrinos.

\begin{tabular}{|c|c|c|c|c|c|c|c|c|}
\hline & $u$ & $d$ & $c$ & $S$ & $t$ & $b$ & $\ell$ & $\nu$ \\
\hline$Q$ & $\frac{2}{3}$ & $-\frac{1}{3}$ & $\frac{2}{3}$ & $-\frac{1}{3}$ & $\frac{2}{3}$ & $-\frac{1}{3}$ & -1 & 0 \\
\hline$I_{3}$ & $\frac{1}{2}$ & $-\frac{1}{2}$ & $\frac{1}{2}$ & $-\frac{1}{2}$ & $\frac{1}{2}$ & $-\frac{1}{2}$ & $-\frac{1}{2}$ & $\frac{1}{2}$ \\
\hline
\end{tabular}

$Z$-boson propagator. Therefore, the interaction vertex between dark bosons and neutrons in Fig. 5 have the following Lorentz structure $i e \bar{\psi}_{n} \gamma^{\mu}\left(a_{f}+b_{f} \gamma^{5}\right) \psi_{n}$ with

$$
\begin{gathered}
a_{f}=Q \varepsilon_{\gamma}+\frac{1}{\sin 2 \theta_{W}}\left(I_{3}-2 Q \sin ^{2} \theta_{W}\right) \tilde{\varepsilon}_{Z}, \\
b_{f}=-\frac{I_{3}}{\sin 2 \theta_{W}} \tilde{\varepsilon}_{Z},
\end{gathered}
$$

where

$\tilde{\varepsilon}_{Z}=\frac{\varepsilon_{Z}+\varepsilon_{\gamma} \tan \theta_{W}\left(m_{V}^{2} / m_{Z}^{2}\right)}{\left(1-m_{V}^{2} / m_{Z}^{2}\right)^{2}+\Gamma_{Z}^{2} / m_{Z}^{2}}\left(1-\frac{m_{V}^{2}}{m_{Z}^{2}}-i \frac{\Gamma_{Z}}{m_{Z}}\right)$,

and $\Gamma_{Z}$ is the $Z$ boson decay width, $Q$ and $I_{3}$ are the electric charge and the weak isospin respectively. In Table I, we list $Q$ and $I_{3}$ for various particles. The values for neutrons can be obtained by summing the corresponding quantum numbers of three quarks $u d d$ in the low energy limit.

Mixing parameters $\varepsilon_{\gamma}$ and $\tilde{\varepsilon}_{Z}$ are responsible for electromagnetic and $\mathrm{NC}$ interactions, respectively. Electromagnetic interaction does not contribute to $\sigma_{\chi n}$ since $Q=0$ for neutrons. On the other hand $\tilde{\varepsilon}_{Z}$ has a feeble dependence on $\varepsilon_{\gamma}$ with a suppression factor $m_{V}^{2} / m_{Z}^{2}$ when $m_{V} \ll m_{Z}$. This explains why $\sigma_{\chi n}$ is still nonzero when $\varepsilon_{Z}=0(\eta=\infty)$.

The spin-averaged $\chi n$ scattering amplitude is given by

$$
\begin{aligned}
\overline{\left|\mathcal{M}_{\chi n}\right|^{2}}= & \frac{8 \pi \alpha_{\chi}}{\left(t-m_{V}^{2}\right)^{2}}\left\{-4 m_{n}^{2}\left[\left(b_{f}^{2}-a_{f}^{2}\right) m_{\chi}^{2}+a_{f}^{2} u\right.\right. \\
& \left.+b_{f}^{2}(s+u)\right]+2\left(a_{f}^{2}+3 b_{f}^{2}\right) m_{\chi}^{4} \\
& -4 a_{f}^{2} u m_{n}^{2}+a_{f}^{2}\left(t^{2}+2 t u+2 u^{2}\right) \\
& \left.+2\left(a_{f}^{2}-b_{f}^{2}\right) m_{n}^{4}+b_{f}^{2}\left(s^{2}+u^{2}\right)\right\},
\end{aligned}
$$

where $s, t$, and $u$ are the Mandelstam variables. DM scatters with neutrons when its velocity is boosted to $0.3 c-0.6 c$ by the NS gravity. It must be treated relativistically. However, neutrons can be treated as at rest since the chemical potential is $\mathcal{O}(200) \mathrm{MeV}$ in the star.
Therefore, from the method in Ref. [82], we are able to write down the DM-neutron scattering cross section as

$\sigma_{\chi n}=\frac{1}{16 \pi \lambda^{1 / 2}\left(s, m_{1}^{2}, m_{2}^{2}\right) \lambda^{1 / 2}\left(s, m_{3}^{2}, m_{4}^{2}\right)} \int_{t_{-}}^{t_{+}} \overline{\left|\mathcal{M}_{\chi n}\right|^{2}} d t$,

where

$$
\lambda(x, y, z)=x^{2}+y^{2}+z^{2}-2 x y-2 y z+2 x z
$$

is the Källén function,

$$
\begin{aligned}
t_{ \pm}= & \frac{1}{2} \sum_{i=1}^{4} m_{i}^{2}-\frac{s}{2}-\frac{1}{2 s}\left(m_{1}^{2}-m_{2}^{2}\right)\left(m_{3}^{2}-m_{4}^{2}\right) \\
& \pm \frac{\lambda^{1 / 2}\left(s, m_{1}^{2}, m_{2}^{2}\right) \lambda^{1 / 2}\left(s, m_{3}^{2}, m_{4}^{2}\right)}{2 s}
\end{aligned}
$$

and

$$
s=m_{1}^{2}+m_{2}^{2}+2 E_{1} m_{2},
$$

where $m_{1}=m_{3}=m_{\chi}$ and $m_{2}=m_{4}=m_{n}$ for the $\chi n$ scattering. In Eq. (A10), the energy $E_{1}=\gamma m_{1}$ is the total energy carried by particle one, which is DM.

\section{Pauli blocking in the $\chi n$ scattering}

Note that if the momentum transfer $\sqrt{-t}$ in Eq. (A7) is smaller than the Fermi momentum, the suppression by Pauli blocking takes effect. We include this in the numerical calculation by incorporating the method in Ref. [68]. Our result agrees with Ref. [68] in the three benchmark scenarios that $\overline{\left|\mathcal{M}_{\chi n}\right|^{2}}$ are constant, $t$-dependent and $t^{2}$ dependent.

\section{Axial-vector contribution in the NR limit}

If $\chi$ can be treated nonrelativistically as well, we have $s=m_{\chi}^{2}+m_{n}^{2}+2 m_{\chi} m_{n}, u=m_{\chi}^{2}+m_{n}^{2}-2 m_{\chi} m_{n}$ and $t=0$. Therefore the amplitude and the cross section become,

$$
\overline{\left|\mathcal{M}_{\chi n}^{\mathrm{NR}}\right|^{2}}=\frac{64 \pi a_{f}^{2} \alpha_{\chi} m_{\chi}^{2} m_{n}^{2}}{m_{V}^{4}}
$$

and

$$
\sigma_{\chi n}^{\mathrm{NR}}=\frac{4 a_{f}^{2} \alpha_{\chi}}{m_{V}^{4}} \frac{m_{\chi}^{2} m_{n}^{2}}{\left(m_{\chi}+m_{n}\right)^{2}}
$$

which are independent of $b_{f}$ where it determines the strength of axial-vector coupling. 


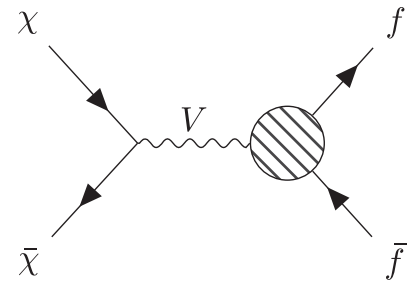

(a) To SM particles
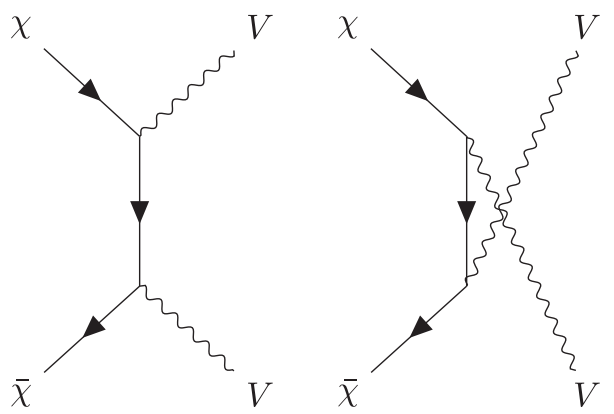

(b) To dark bosons

FIG. 7. Various channels for DM annihilation

\section{APPENDIX B: DM ANNIHILATION}

We can divide the DM annihilation into two categories, which are $m_{V} \geq m_{\chi}$ and $m_{V}<m_{\chi}$, respectively. For the prior case, DM can only annihilate into SM particles as shown in Fig. 7(a). For the later one, as long as $g_{d} \gg e \varepsilon_{\gamma, Z}$, the dominant annihilation products are two dark bosons $V$ as shown in Fig. 7(b). The amplitude for $\chi \bar{\chi} \rightarrow f \bar{f}$ is given by

$$
\begin{aligned}
\overline{\left|\mathcal{M}_{\chi \bar{\chi} \rightarrow f \bar{f}}\right|^{2}=} & \frac{8 \pi \alpha_{\chi}}{\left(s-m_{V}^{2}\right)^{2}+m_{V}^{2} \Gamma_{V}^{2}}\left\{a_{f}^{2}\left[-2\left(m_{f}^{2}+m_{\chi}^{2}\right)\left(-m_{f}^{2}-m_{\chi}^{2}+2 u\right)+s^{2}+2 s u+2 u^{2}\right]\right. \\
& \left.+b_{f}^{2}\left[-4 m_{\chi}^{2}\left(m_{f}^{2}+t+u\right)-2 m_{f}^{4}+6 m_{\chi}^{4}+t^{2}+u^{2}\right]\right\},
\end{aligned}
$$

where $\Gamma_{V}$ is the $V$ decay width. Assuming DM is at rest in the star, the amplitude can be simplified into

$$
\overline{\left|\mathcal{M}_{\chi \bar{\chi} \rightarrow f \bar{f}}\right|^{2}}=\frac{128 \pi \alpha_{\chi}}{\left(4 m_{\chi}^{2}-m_{V}^{2}\right)^{2}+m_{V}^{2} \Gamma_{V}^{2}} m_{\chi}^{4}\left[a_{f}^{2}\left(1+\frac{1}{2} \frac{m_{f}^{2}}{m_{\chi}^{2}}\right)+b_{f}^{2}\left(1-\frac{m_{f}^{2}}{m_{\chi}^{2}}\right)\right] .
$$

The partial decay widths of $V$ are given by

$$
\Gamma_{V}=\frac{m_{V}}{12 \pi} \sqrt{1-4 \frac{m_{f}^{2}}{m_{V}^{2}}}\left[a_{f}^{2}\left(1+2 \frac{m_{f}^{2}}{m_{V}^{2}}\right)+b_{f}^{2}\left(1-4 \frac{m_{f}^{2}}{m_{V}^{2}}\right)\right]
$$

for $V \rightarrow f \bar{f}$ and

$$
\Gamma_{V}=\frac{\alpha_{\chi}}{3} m_{V} \sqrt{1-4 \frac{m_{\chi}^{2}}{m_{V}^{2}}}\left(1+2 \frac{m_{\chi}^{2}}{m_{V}^{2}}\right)
$$

for $V \rightarrow \chi \bar{\chi}$. Note that we have omitted the Heaviside theta function $\theta\left(m_{V}-2 m_{\chi, f}\right)$ in the above expressions but it is always implemented when we perform the calculation to ensure the energy conservation. Besides, when $m_{\chi}>m_{V}$, the channel $\chi \bar{\chi} \rightarrow 2 V$ is allowed and the amplitude is

$$
\begin{aligned}
\overline{\left|\mathcal{M}_{\chi \bar{\chi} \rightarrow 2 V}\right|^{2}}= & -\frac{32 \pi^{2} \alpha_{\chi} 2}{\left(t-m_{\chi}^{2}\right)^{2}\left(u-m_{\chi}^{2}\right)^{2}}\left\{m_{V}^{4}\left[6 m_{\chi}^{2}(t+u)-6 m_{\chi}^{4}+t^{2}-8 t u+u^{2}\right]\right. \\
& +4 m_{V}^{2}\left[m_{\chi}^{4}(t+u)-4 m_{\chi}^{2} t u+t u(t+u)\right]-m_{\chi}^{4}\left(3 t^{2}+14 t u+3 u^{2}\right) \\
& \left.+m_{\chi}^{2}\left(t^{3}+7 t^{2} u+7 t u^{2}+u^{3}\right)+6 m_{\chi}^{2}-t u\left(t^{2}+u^{2}\right)\right\} .
\end{aligned}
$$

In the NR limit,

$$
\overline{\left|\mathcal{M}_{\chi \bar{\chi} \rightarrow 2 V}\right|^{2}}=256 \pi^{2} \alpha_{\chi}^{2} \frac{m_{\chi}^{2}\left(m_{\chi}^{2}-m_{V}^{2}\right)}{\left(m_{V}^{2}-2 m_{\chi}^{2}\right)^{2}}
$$


$\eta=1$

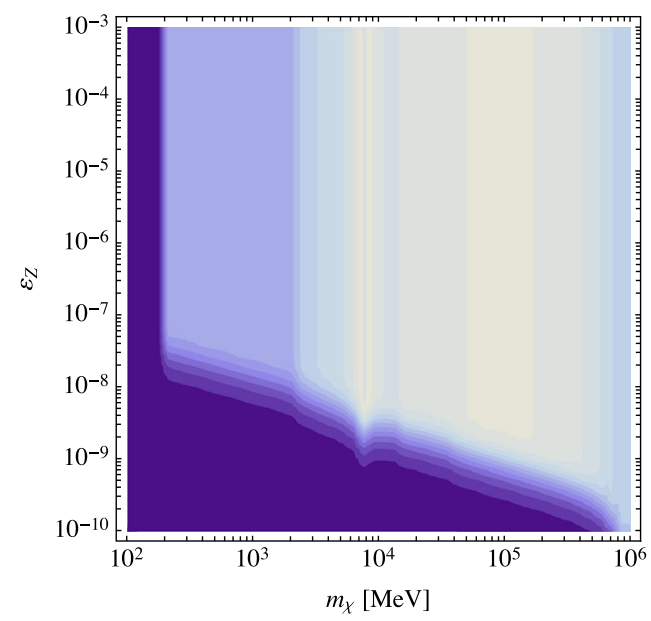

$\eta=\infty$

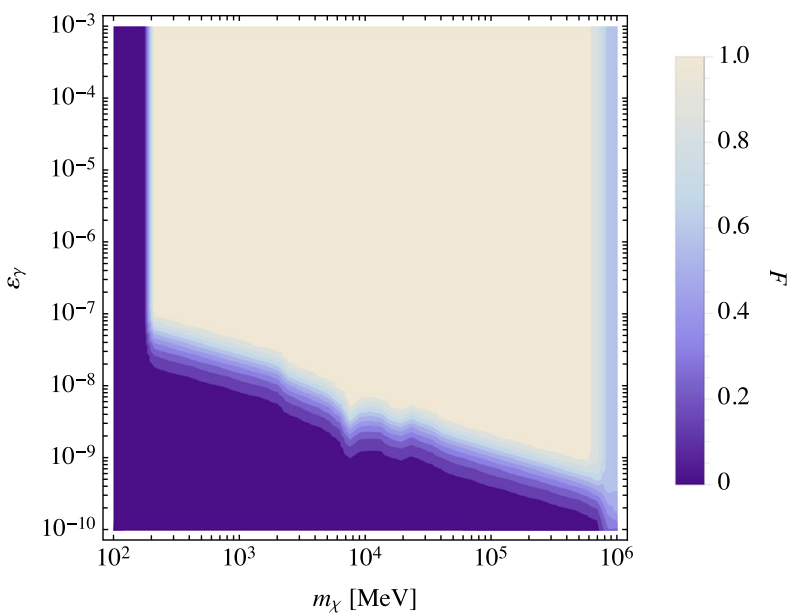

FIG. 8. Fraction $F$ of dark boson decay into SM particles that contribute to the heating effect.

Thus, the general expression for annihilation cross section is obtained by using the Fermi golden rule,

$$
\sigma v=\frac{\overline{|\mathcal{M}|^{2}}}{32 \pi m_{\chi}^{2}} \sqrt{1-\frac{m_{f}^{2}}{m_{\chi}^{2}}} \theta\left(m_{\chi}-\mu_{F}^{f}\right),
$$

where $m_{f}$ is the final state particle mass and $\mu_{F}^{f}$ is the chemical potential of fermion $f$ in the star. There is no chemical potential for dark boson $V$. Therefore, we arrive at

$$
(\sigma v)^{f \bar{f}}=4 \alpha_{\chi} \kappa m_{\chi}^{2} \sqrt{1-\frac{m_{f}^{2}}{m_{\chi}^{2}}} \theta\left(m_{\chi}-\mu_{F}^{f}\right),
$$

where

$\kappa=\frac{1}{\left(4 m_{\chi}^{2}-m_{V}^{2}\right)^{2}+m_{V}^{2} \Gamma_{V}^{2}}\left[a_{f}^{2}\left(1+\frac{1}{2} \frac{m_{f}^{2}}{m_{\chi}^{2}}\right)+b_{f}^{2}\left(1-\frac{m_{f}^{2}}{m_{\chi}^{2}}\right)\right]$

for $\chi \bar{\chi} \rightarrow f \bar{f}$ and

$$
(\sigma v)^{2 V}=8 \pi \alpha_{\chi}^{2} \sqrt{1-\frac{m_{V}^{2}}{m_{\chi}^{2}}} \frac{\left(m_{\chi}^{2}-m_{V}^{2}\right)}{\left(m_{V}^{2}-2 m_{\chi}^{2}\right)^{2}}
$$

for $\chi \bar{\chi} \rightarrow 2 V$. The total annihilation cross section is the sum of both

$$
\sigma v=(\sigma v)^{f \bar{f}}+(\sigma v)^{2 V}
$$

We note that the second term contributes when $m_{V}<m_{\chi}$.

\section{APPENDIX C: DARK BOSON IN THE STAR}

Dark bosons can be produced from DM annihilation once $m_{V}<m_{\chi}$. This channel is thought to have feeble effect on the heating since $V$ interacts weakly with the NS medium and escapes without any trace. However, we found that, depending on the strength of $\varepsilon_{\gamma, Z}, V$ can decay into SM particles before it reaches the surface of the star. In the case that the decay length $\ell_{\mathrm{dec}}$ is much smaller than the radius of the star, the total energy released from the annihilation can be fully deposited to the star. (See Fig. 1(a).) We also examine the case where $V$ is produced in the DM rich region in the center of the star. $V$ could undergo multiple scattering with the surrounding DM and self-trapped until it decays. See Fig. 1(b). This is another way to extract energy from $V$. We discuss both effects in the following.

\section{Decay length}

The dark boson decay length with time dilation effect is given by

$$
\ell_{\mathrm{dec}}=v \gamma \tau_{\mathrm{dec}},
$$

where $v=\sqrt{1-m_{V}^{2} / m_{\chi}^{2}}$ is the $V$ velocity and $\tau_{\mathrm{dec}}=\Gamma_{V}^{-1}$ the $V$ lifetime at rest. Let us assume that $V$ is produced in the center of the star and its propagation distance is $R_{0}$. Figure 8 presents $F$ defined in Eq. (11), i.e., the fraction of $V$ converting into SM particles after traveling a distance $r=R_{0}$, as functions of $\varepsilon_{Z, \gamma}$ and $m_{\chi}$ for $m_{V}=0.1 m_{\chi}$. We have subtracted neutrino contributions from $F$ since they cannot generate heat. Since the branching ratio of $V$ decays to neutrinos is nonzero in the case of $V-Z$ mass mixing, $F$ is generally smaller than 1 for $\eta=1$. For $\eta=\infty$, no neutrinos can be produced, thus $F$ can reach unity.

In these figures, the chemical potential for electron $\mu_{F}^{e}$ is about $\mathcal{O}(170) \mathrm{MeV}$. For a dark boson at rest with $m_{V} \leq \mu_{F}^{e}$, 
$V \rightarrow e^{+} e^{-}$can be Pauli blocked even for $m_{V} \geq 2 m_{e}$. On the other hand, if $V$ is highly boosted as a result of heavy DM annihilation, $V \rightarrow e^{+} e^{-}$is not Pauli blocked as long as $m_{\chi} \geq \mu_{F}^{e}$. Therefore, to enable $V \rightarrow f \bar{f}$ decays, two conditions are required. The first is $m_{\chi} \geq \mu_{F}^{f}$ and the second is $m_{V} \geq 2 m_{f}$.

\section{Dark boson-DM interaction length}

Feynman diagrams contributing to $\chi V$ scattering are shown in Fig. 9 and the amplitude is given by
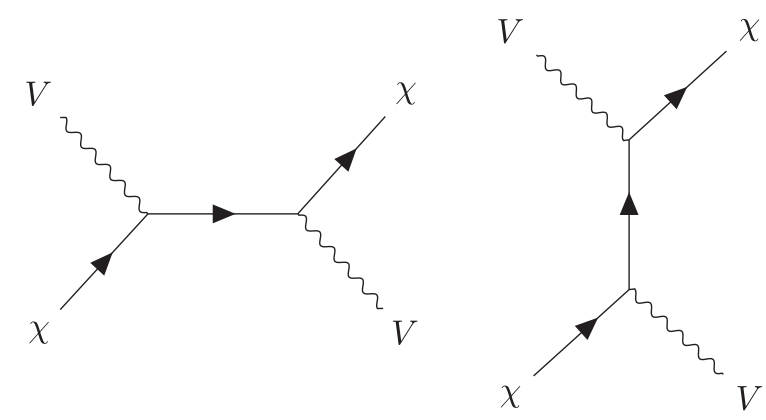

FIG. 9. $\chi V$ scattering via $s$ and $t$ channels.

$$
\begin{aligned}
\overline{\left|\mathcal{M}_{\chi V}\right|^{2}}= & \frac{64 \pi^{2}}{3} \frac{\alpha_{\chi}^{2}}{\left(s-m_{\chi}^{2}\right)^{2}\left(t-m_{\chi}^{2}\right)^{2}}\left\{m_{V}^{4}\left[6 m_{\chi}^{2}(s+t)-6 m_{\chi}^{4}+s^{2}-8 s t+t^{2}\right]\right. \\
& -m_{\chi}^{4}\left(3 s^{2}+14 s t+3 t^{2}\right)+m_{\chi}^{2}\left(s^{3}+7 s^{2} t+7 s t^{2}+t^{3}\right) \\
& \left.+4 m_{V}^{2}\left[m_{\chi}^{4}(s+t)-4 s t m_{\chi}^{2}+s t(s+t)\right]+6 m_{\chi}^{8}-s t\left(s^{2}+t^{2}\right)\right\}
\end{aligned}
$$

To compute the scattering cross section $\sigma_{\chi V}$, it is fair to assume DM at rest. However, $V$ is produced with relativistic velocity since $m_{\chi}>m_{V}$. We follow the procedure given in Eqs. (A7)-(A10) and set $m_{1}=m_{3}=m_{V}$ and $m_{2}=m_{4}=m_{\chi}$. Thus,

$$
\sigma_{\chi V}=\frac{1}{16 \pi \lambda\left(s, m_{V}^{2}, m_{\chi}^{2}\right)} \int_{t_{-}}^{t_{+}} \overline{\left|\mathcal{M}_{\chi V}\right|^{2}} d t .
$$

Note that $\chi V$ scattering is not subject to Pauli blocking since DM does not become degenerate in the presence of annihilation.

The $\chi V$ scattering length $\ell_{\chi V}$ is given by

$$
\ell_{\chi V}=\left(n_{\chi} \sigma_{\chi V}\right)^{-1}
$$

with $n_{\chi} \equiv N_{\chi} / V_{\chi}$ the average DM number density. The volume characterizing DM in NS is $V_{\chi}=4 \pi r_{\text {th }}^{3} / 3$, where

$$
r_{\mathrm{th}} \approx 2.4 \times 10^{3} \mathrm{~cm}\left(\frac{T_{\chi}}{10^{5} \mathrm{~K}} \frac{10 \mathrm{MeV}}{m_{\chi}}\right)^{1 / 2}
$$

is the thermal radius. If $\ell_{\chi V} \ll r_{\text {th }}, V$ can scatter with surrounding DM multiple times and gradually lose its kinetic energy. However, our numerical result shows that $\ell_{\chi V} \gg r_{\text {th }}$ in all of our interested parameter space. In Fig. 10, we take $\alpha_{\chi}=1$ and $T_{\chi}=1000 \mathrm{~K}$. The choice $\alpha_{\chi}<1$ makes $\ell_{\chi V}$ even longer due to a weaker $\chi V$ interaction. For $\eta=1$, the region for $\ell_{\chi V} / r_{\text {th }}<1$ happens when $m_{\chi} \lesssim 300 \mathrm{MeV}$. However, even $V$ can be $\eta=1$

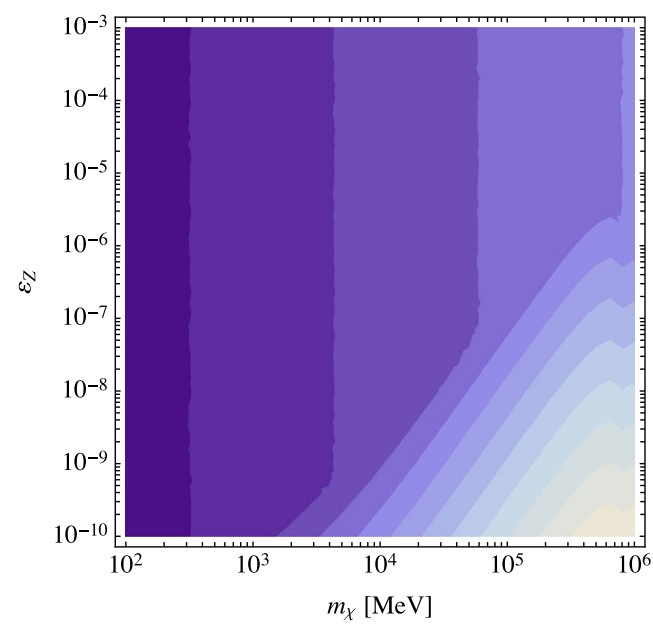

$\eta=\infty$

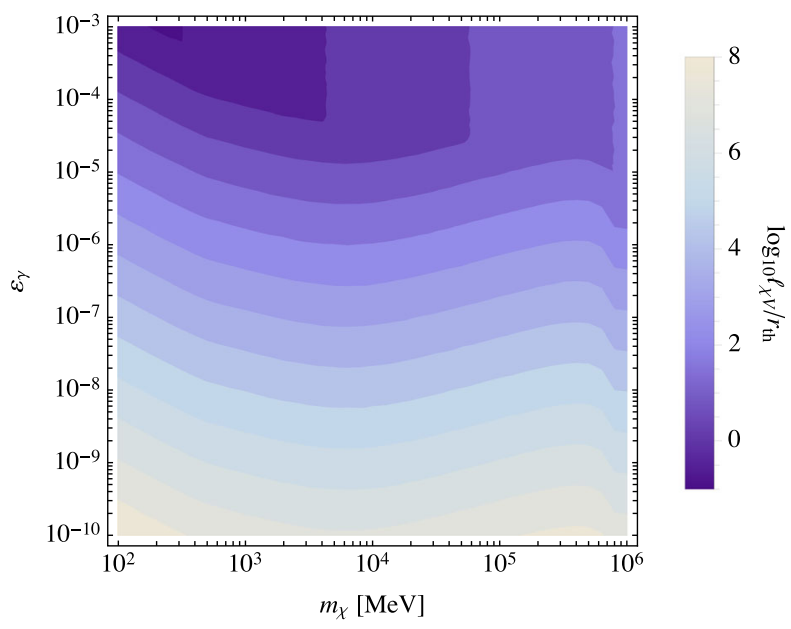

FIG. 10. The ratio $\ell_{\chi V} / r_{\text {th }}$ for $\eta=1$ and $\infty$. We take $\alpha_{\chi}=1$ and $T_{\chi}=1000 \mathrm{~K}$ in the calculation. 
self-trapped; it hardly decays into particles other than neutrinos because the allowed channels, eg. $e^{ \pm}$and $\mu^{ \pm}$, are Pauli blocked. For $\eta=\infty$, only a very small parameter space leads to $\ell_{\chi V} / r_{\text {th }}<1$. Therefore, we conclude that the self-trapping of $V$ is insignificant; hence only $V$ decays contribute to the energy injection.
[1] G. Aad et al. (ATLAS Collaboration), Eur. Phys. J. C 75, 299 (2015); 75, 408(E) (2015).

[2] J. Abdallah et al., Phys. Dark Universe 9-10, 8 (2015).

[3] J. Aalbers et al. (DARWIN Collaboration), J. Cosmol. Astropart. Phys. 11 (2016) 017.

[4] D. S. Akerib et al. (LUX Collaboration), Phys. Rev. Lett. 118, 021303 (2017).

[5] C. Amole et al. (PICO Collaboration), Phys. Rev. Lett. 118, 251301 (2017).

[6] D. S. Akerib et al. (LUX Collaboration), Phys. Rev. Lett. 118, 251302 (2017).

[7] E. Aprile et al. (XENON Collaboration), Phys. Rev. Lett. 119, 181301 (2017).

[8] E. Aprile et al. (XENON Collaboration), Phys. Rev. Lett. 121, 111302 (2018).

[9] E. Aprile et al. (XENON Collaboration), Phys. Rev. Lett. 123, 241803 (2019).

[10] E. Aprile et al. (XENON Collaboration), Phys. Rev. Lett. 123, 251801 (2019).

[11] M. G. Aartsen et al. (IceCube PINGU Collaboration), arXiv:1401.2046.

[12] K. Choi et al. (Super-Kamiokande Collaboration), Phys. Rev. Lett. 114, 141301 (2015).

[13] M. G. Aartsen et al. (IceCube Collaboration), Eur. Phys. J. C 77, 146 (2017).

[14] M. Aguilar et al. (AMS Collaboration), Phys. Rev. Lett. 115, 211101 (2015).

[15] M. Ackermann et al. (Fermi-LAT Collaboration), Astrophys. J. 840, 43 (2017).

[16] G. Ambrosi et al. (DAMPE Collaboration), Nature (London) 552, 63 (2017).

[17] G. Beck, M. Kumar, E. Malwa, B. Mellado, and R. Temo, arXiv:2102.10596.

[18] C. Kouvaris, Phys. Rev. D 77, 023006 (2008).

[19] A. de Lavallaz and M. Fairbairn, Phys. Rev. D 81, 123521 (2010).

[20] C. Kouvaris and P. Tinyakov, Phys. Rev. D 82, 063531 (2010).

[21] M. Baryakhtar, J. Bramante, S. W. Li, T. Linden, and N. Raj, Phys. Rev. Lett. 119, 131801 (2017).

[22] N. Raj, P. Tanedo, and H. B. Yu, Phys. Rev. D 97, 043006 (2018).

[23] C. S. Chen and Y. H. Lin, J. High Energy Phys. 08 (2018) 069.

[24] N. F. Bell, G. Busoni, and S. Robles, J. Cosmol. Astropart. Phys. 09 (2018) 018.

[25] J. F. Acevedo, J. Bramante, R. K. Leane, and N. Raj, J. Cosmol. Astropart. Phys. 03 (2020) 038.

[26] A. Joglekar, N. Raj, P. Tanedo, and H. B. Yu, Phys. Lett. B 809, 135767 (2020).
[27] W. Y. Keung, D. Marfatia, and P. Y. Tseng, J. High Energy Phys. 07 (2020) 181.

[28] A. Joglekar, N. Raj, P. Tanedo, and H. B. Yu, Phys. Rev. D 102, 123002 (2020).

[29] B. Dasgupta, A. Gupta, and A. Ray, J. Cosmol. Astropart. Phys. 10 (2020) 023.

[30] R. Garani, A. Gupta, and N. Raj, Phys. Rev. D 103, 043019 (2021).

[31] C. Kouvaris and P. Tinyakov, Phys. Rev. D 83, 083512 (2011).

[32] S. C. Leung, M. C. Chu, and L. M. Lin, Phys. Rev. D 84, 107301 (2011).

[33] C. Kouvaris, Phys. Rev. Lett. 108, 191301 (2012).

[34] S. D. McDermott, H. B. Yu, and K. M. Zurek, Phys. Rev. D 85, 023519 (2012).

[35] T. Güver, A. E. Erkoca, M. Hall Reno, and I. Sarcevic, J. Cosmol. Astropart. Phys. 05 (2014) 013.

[36] J. Bramante, K. Fukushima, and J. Kumar, Phys. Rev. D 87, 055012 (2013).

[37] J. Bramante, K. Fukushima, J. Kumar, and E. Stopnitzky, Phys. Rev. D 89, 015010 (2014).

[38] C. Kouvaris and P. Tinyakov, Phys. Rev. D 90, 043512 (2014).

[39] M. I. Gresham and K. M. Zurek, Phys. Rev. D 99, 083008 (2019).

[40] B. Grinstein, C. Kouvaris, and N. G. Nielsen, Phys. Rev. Lett. 123, 091601 (2019).

[41] R. Garani, Y. Genolini, and T. Hambye, J. Cosmol. Astropart. Phys. 05 (2019) 035.

[42] G. L. Lin and Y. H. Lin, J. Cosmol. Astropart. Phys. 08 (2020) 022.

[43] A. Nelson, S. Reddy, and D. Zhou, J. Cosmol. Astropart. Phys. 07 (2019) 012.

[44] J. Ellis, G. Hütsi, K. Kannike, L. Marzola, M. Raidal, and V. Vaskonen, Phys. Rev. D 97, 123007 (2018).

[45] A. Bauswein, G. Guo, J. H. Lien, Y. H. Lin, and M. R. Wu, arXiv:2012.11908.

[46] R. K. Leane, T. Linden, P. Mukhopadhyay, and N. Toro, Phys. Rev. D 103, 075030 (2021).

[47] S. Tulin and H. B. Yu, Phys. Rep. 730, 1 (2018).

[48] S. W. Randall, M. Markevitch, D. Clowe, A. H. Gonzalez, and M. Bradac, Astrophys. J. 679, 1173 (2008).

[49] M. G. Walker and J. Penarrubia, Astrophys. J. 742, 20 (2011).

[50] M. Boylan-Kolchin, J. S. Bullock, and M. Kaplinghat, Mon. Not. R. Astron. Soc. 415, L40 (2011).

[51] M. Boylan-Kolchin, J. S. Bullock, and M. Kaplinghat, Mon. Not. R. Astron. Soc. 422, 1203 (2012).

[52] O. D. Elbert, J. S. Bullock, S. Garrison-Kimmel, M. Rocha, J. Oñorbe, and A. H. Peter, Mon. Not. R. Astron. Soc. 453, 29 (2015). 
[53] T. Lin, H. B. Yu, and K. M. Zurek, Phys. Rev. D 85, 063503 (2012).

[54] B. Holdom, Phys. Lett. 166B, 196 (1986).

[55] P. Galison and A. Manohar, Phys. Lett. 136B, 279 (1984).

[56] R. Foot, Int. J. Mod. Phys. D 13, 2161 (2004).

[57] D. Feldman, B. Kors, and P. Nath, Phys. Rev. D 75, 023503 (2007).

[58] N. Arkani-Hamed, D. P. Finkbeiner, T. R. Slatyer, and N. Weiner, Phys. Rev. D 79, 015014 (2009).

[59] M. Pospelov and A. Ritz, Phys. Lett. B 671, 391 (2009).

[60] K. S. Babu, C. F. Kolda, and J. March-Russell, Phys. Rev. D 57, 6788 (1998).

[61] H. Davoudiasl, H. S. Lee, and W. J. Marciano, Phys. Rev. D 85, 115019 (2012).

[62] H. Davoudiasl, H. S. Lee, I. Lewis, and W. J. Marciano, Phys. Rev. D 88, 015022 (2013).

[63] For various mass generation mechanisms in the dark sector, see for example, N. F. Bell, Y. Cai, and R. K. Leane, J. Cosmol. Astropart. Phys. 01 (2017) 039.

[64] N. F. Bell, Y. Cai, and R. K. Leane, J. Cosmol. Astropart. Phys. 08 (2016) 001.

[65] M. Duerr, F. Kahlhoefer, K. Schmidt-Hoberg, T. Schwetz, and S. Vogl, J. High Energy Phys. 09 (2016) 042.

[66] J. P. Gardner et al., Space Sci. Rev. 123, 485 (2006).

[67] W. Skidmore et al. (TMT International Science Development Teams \& TMT Science Advisory Committee), Res. Astron. Astrophys. 15, 1945 (2015).

[68] N. F. Bell, G. Busoni, S. Robles, and M. Virgato, J. Cosmol. Astropart. Phys. 09 (2020) 028.
[69] N. F. Bell, G. Busoni, S. Robles, and M. Virgato, J. Cosmol. Astropart. Phys. 03 (2021) 086.

[70] G. Steigman, B. Dasgupta, and J. F. Beacom, Phys. Rev. D 86, 023506 (2012).

[71] J. Bramante, A. Delgado, and A. Martin, Phys. Rev. D 96, 063002 (2017).

[72] B. Dasgupta, A. Gupta, and A. Ray, J. Cosmol. Astropart. Phys. 08 (2019) 018.

[73] C. Ilie, J. Pilawa, and S. Zhang, Phys. Rev. D 102, 048301 (2020).

[74] A. Sung, H. Tu, and M. R. Wu, Phys. Rev. D 99, 121305 (2019).

[75] A. Sung, G. Guo, and M. R. Wu, Phys. Rev. D 103, 103005 (2021).

[76] E. M. Riordan, M. W. Krasny, K. Lang, P. De Barbaro, A. Bodek, S. Dasu, N. Varelas, X. Wang, R. G. Arnold, D. Benton et al., Phys. Rev. Lett. 59, 755 (1987).

[77] A. Bross, M. Crisler, S. H. Pordes, J. Volk, S. Errede, and J. Wrbanek, Phys. Rev. Lett. 67, 2942 (1991).

[78] M. Abdullah, J. B. Dent, B. Dutta, G. L. Kane, S. Liao, and L. E. Strigari, Phys. Rev. D 98, 015005 (2018).

[79] M. Cirelli, P. Panci, K. Petraki, F. Sala, and M. Taoso, J. Cosmol. Astropart. Phys. 05 (2017) 036.

[80] S. Cassel, J. Phys. G 37, 105009 (2010).

[81] JWST Pocket Guide, June 2021, https://www.stsci.edu/files/ live/sites/www/files/home/jwst/instrumentation/ _documents/jwst-pocket-guide.pdf.

[82] V. Ilisie, Concepts in Quantum Field Theory (Springer, New York, 2016). 